\title{
Severe fever with thrombocytopenia syndrome (SFTS) treated with a novel antiviral medication, favipiravir (T-705)
}

\author{
Rui Song ${ }^{1} \cdot$ Zhihai Chen ${ }^{1} \cdot$ Wei $\mathrm{Li}^{2}$ (1)
}

Received: 26 June 2019 / Accepted: 1 October 2019 / Published online: 31 October 2019

c) Springer-Verlag GmbH Germany, part of Springer Nature 2019

\begin{abstract}
Background Severe fever and thrombocytopenia syndrome (SFTS) is an acute illness with a high mortality (16.2-29.1\%). Unfortunately, there is no specific cure or vaccine for SFTS.

Methods In this open-label study, two patients with SFTS were treated with favipiravir, a new antiviral drug.

Results Patients had a sustainable virologic, immunologic and symptomatic recovery.

Conclusions Favipiravir may be a prosiming drug for the treatment of SFTS.
\end{abstract}

Keywords Severe fever and thrombocytopenia syndrome $\cdot$ SFTS $\cdot$ Favipiravir

\section{Introduction}

Severe fever and thrombocytopenia syndrome (SFTS) is caused by a novel bunyavirus: severe fever and thrombocytopenia syndrome virus (SFTSV). Cases of infection have been reported in central and northeastern China, South Korea, and southern Japan. The most recent updates on the cumulative reported cases are 7419 in China by 2016, 335 in South Korea by 2016, and 319 in Japan by 2017 [1, 2]. SFTSV-like viruses-such as the Heartland virus, Hunter Island virus, and Malsoor virus-continue to be isolated worldwide [3, 4].

SFTS is an acute illness, with a reported mortality rate of $16.2-29.1 \%[1,2]$. Patients with SFTS usually suffer fever, severe haemorrhagic, digestive, or neurologic symptoms,

Electronic supplementary material The online version of this article (https://doi.org/10.1007/s15010-019-01364-9) contains supplementary material, which is available to authorized users.

Zhihai Chen

zhccmu@sina.com

$\triangle$ Wei Li

vision988@126.com

1 Center of Infectious Disease, Beijing Ditan Hospital, Capital Medical University, 8 Jingshun East Street, Chaoyang District, 100015 Beijing, People's Republic of China

2 Cancer Center, Beijing Ditan Hospital, Capital Medical University, 8 Jingshun East Street, Chaoyang District, 100015 Beijing, People's Republic of China and can die from multi-organ failure [5, 6]. Currently, there is no curative treatment, specific antiviral medication, or vaccine against the SFTSV.

Favipiravir (T-705, Avigan) is an antiviral drug targeting many RNA viruses. It can inhibit viral RNA-dependent RNA polymerase selectively or induce RNA transversion mutations to produce a nonviable viral phenotype [7]. Its activity has been demonstrated in the laboratory setting against influenza viruses, yellow fever virus, hand-foot-and-mouth disease virus, bunyavirus, SFTSV, and several other viruses $[8,9]$. A clinical trial investigating favipiravir against the Ebola virus showed that it could reduce mortality from 30 to $15 \%$ [10]. Favipiravir can be used safely in humans and has been approved to treat influenza in Japan. It does not inhibit mammalian RNA or DNA synthesis and is not toxic to mammalian cells [6]. Reports have indicated that favipiravir has a better safety profile compared with ribavirin [11].

The activity of favipiravir against the SFTSV and other Bunyaviruses has been demonstrated in several studies using small animals. In mice infected with the SFTSV, the serum viral load was undetectable 2 days after favipiravir use. In another study using Bunyavirus-infected mice, the favipiravir-treated group had no deaths, whereas the ribavirin-treated group suffered many deaths [12], thereby demonstrating the potential protective effect of favipiravir against the SFTSV.

Reports focusing on favipiravir use in patients infected with the SFTSV are lacking. Here, we report two patients with SFTS treated with this novel antiviral medication who achieved complete recovery. 


\section{Methods}

Both patients with SFTS were treated at our hospital with favipiravir orally for a length of 5 days, with a loading dose of $1600 \mathrm{mg}$ twice a day; from day 2 to day 5, $600 \mathrm{mg}$ was given twice a day. Both patients in this study were informed about the potential benefits and risks of using Favipiravir, and both agreed to use it and signed informed consent.

Favipiravir is licensed and approved in Japan to treat influenza viral infections. In China, favipiravir tablets were approved to begin clinical trials by the China Food and Drug Administration in 2016. In 2018, we received a major grant from the National Science and Technology Major Projects for Major New Drugs Innovation and Development, for 'Clinical Research on Anti-severe fever thrombocytopenia syndrome bunyavirus Drugs' (No. 2018ZX09711003-014-003).

\section{Results}

\section{Patient 1}

Patient 1 was a 38-year-old male who came to our hospital with a chief complaint of fever $\left(39.3{ }^{\circ} \mathrm{C}\right)$ lasting 3 days without a clear origin. He also complained of severe malaise, chills, headache, joint pain in the lower extremities, and progressive fatigue. Coughing, vomiting, heavy sweating, abdominal pain, diarrhoea, skin rash, weight loss, or disturbance of consciousness was not observed.

A physical examination revealed no abnormality. Laboratory tests revealed the following levels: haemoglobin (Hb) $133 \mathrm{~g} / \mathrm{L}$, alanine transaminase (ALT) 54.1 U/L, and creatine kinase (CK) $354 \mathrm{U} / \mathrm{L}$, white blood cell (WBC) count $4.3 \times 10^{9} / \mathrm{L}$, and platelet count $121 \times 10^{9} / \mathrm{L}$. Renalfunction tests were within normal ranges.

The patient had been hepatitis B virus (HBV)-positive for more than a decade and had never sought treatment. The HBV viral load was $\sim 10^{3} \mathrm{IU} / \mathrm{mL}$ upon hospital admission. Abnormal liver function or symptoms in the digestive system, or other serious illnesses, had not been reported in the past.

The patient's mother had suffered a tick bite 1 month previously in Hubei Province, China (which is an SFTS epidemic area). She was diagnosed with SFTS, and our patient returned to Hubei Province to take care of her 3 weeks earlier. Sadly, his mother died 2 weeks later.

Upon hospital admission, blood tests showed a decreased platelet count of $107 \times 10^{9} / \mathrm{L}$. A nucleic-acid test confirmed the diagnosis of SFTS, with a viral load of
$4.1 \times 10^{3}$ copies/mL (Fig. 1). The patient suffered a high body temperature of $38.9{ }^{\circ} \mathrm{C}$. Despite empirical use of doxycycline (0.1 g, b.i.d., p.o.) and aspirin-DL-lysine (i.v.), his body temperature increased to $39.3{ }^{\circ} \mathrm{C}$ with aggravated headache, fatigue, and joint pain in the lower extremities.

The patient was given favipiravir (p.o.) on the night of day 4 of his disease course (day 2 after hospital admission). The initial dose of favipiravir was $1600 \mathrm{mg}$ (p.o.). The same dose was given the next morning (day 5). A reduced dose of $600 \mathrm{mg}$ was given q12 (p.o.) starting from the night of day 5 and continuing for another 4 days (until the night of day 9).

At the beginning of the treatment course, the viral load peaked at $3.99 \times 10^{4}$ copies $/ \mathrm{mL}$ at day 2 of favipiravir treatment. It became undetectable sharply at day 4 , and stayed undetectable even after ceasing favipiravir (Fig. 1). His body temperature returned to normal at day 3 of favipiravir treatment. The platelet count continued to decrease, reaching the nadir of $71.8 \times 10^{9} / \mathrm{L}$ at day 7 (day 4 of favipiravir treatment), after which it started to increase. A reduced lymphocyte count was observed during the disease course and recovered within 2 days of favipiravir use. Liver function started to recover at day 11 of the disease course. His symptoms disappeared at day 8 (day 5 of favipiravir treatment) (Supplementary Fig. 1). The HBV viral load was stable throughout the treatment course. The patient was discharged shortly afterward. He returned to us after 4 months for follow-up, and all test results were within normal ranges.

\section{Patient 2}

Patient 2 was a 48-year-old male, admitted at 11 days after the onset of fever, with melena for 2 days and drowsiness for $10 \mathrm{~h}$. Eleven days previously, the patient had a fever of $38.9{ }^{\circ} \mathrm{C}$ along with fatigue and nausea, but without vomiting, chills, sore throat, cough, joint pain, diarrhoea or other symptoms. He had not responded to antibiotics (ceftriaxone) at local clinics. At day 7, the patient started to suffer periumbilical abdominal pain and was transferred to a major local hospital. An abdominal ultrasound revealed no abnormality. A qualitative nucleic-acid test against the SFTSV reported positive. The patient was treated with ribavirin, but his symptoms worsened rapidly, and he started to show anorexia, abdominal distention, melena (3-4 times per day), mental sluggishness, worsening fatigue, reluctance to speak, disorientation and drowsiness at day 10. Petechiae were seen at the puncture site of the intramuscular injection.

Before hospital admission, blood tests showed that the platelet count had dropped from $107 \times 10^{9} / \mathrm{L}$ to $31 \times 10^{9} / \mathrm{L}$. The WBC count had dropped from $4.45 \times 10^{9} / \mathrm{L}$ to $2.3 \times 10^{9} / \mathrm{L}$. The level of ALT increased gradually to 139 $\mathrm{U} / \mathrm{L}$, and that of aspartate transaminase (AST) to $444 \mathrm{U} / \mathrm{L}$. 

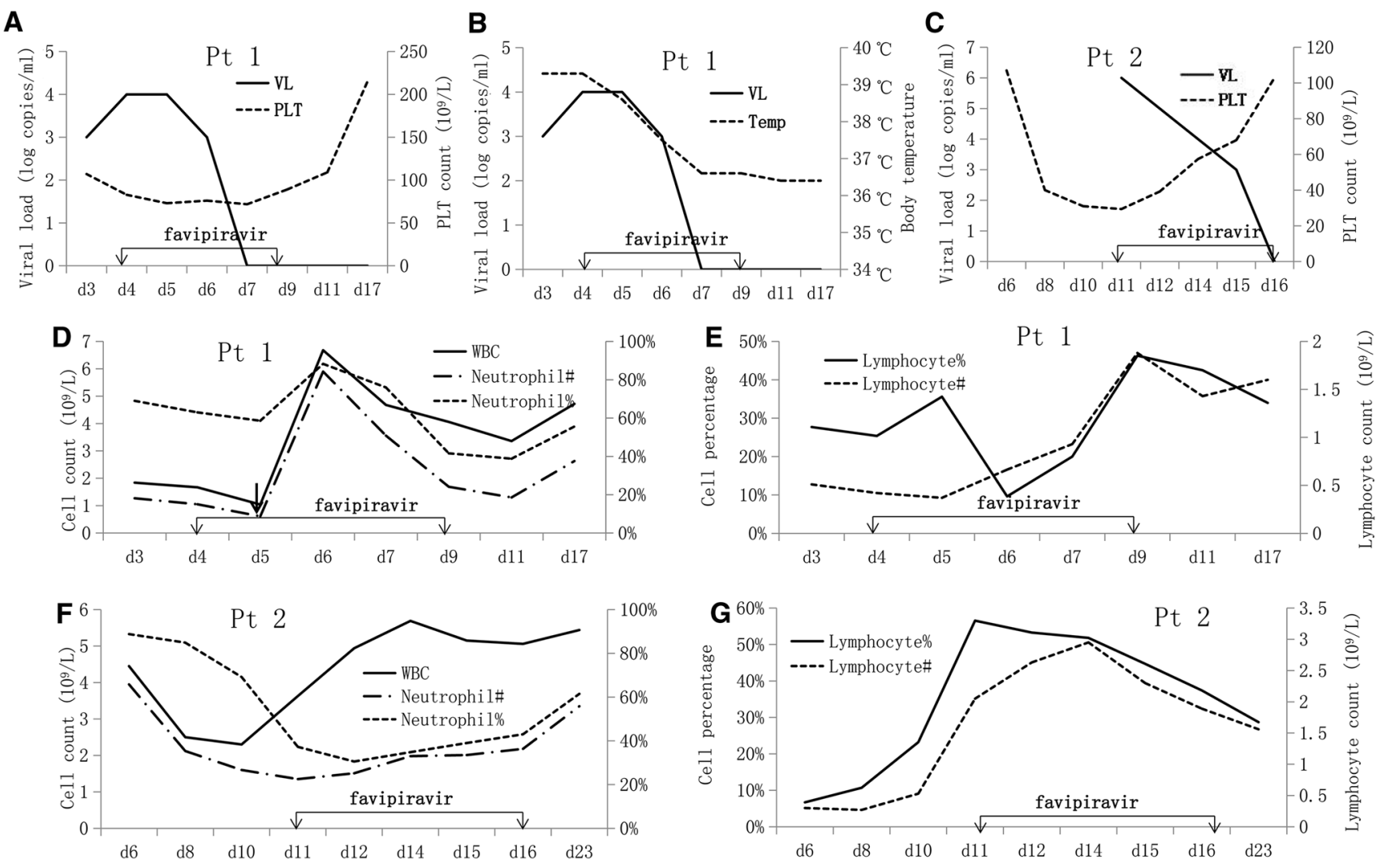

Fig. 1 Dynamic changes of viral load, body temperature, and blood cell counts before and after favipiravir treatment. Viral load and platelet count changes are shown in patient 1 (a) and patient 2 (c). Viral load and body temperature change in patient 1 (b). White blood cell

count, neutrophil count, and percentage changes in patient 1 (d) and patient 2 (f). Lymphocyte count and percentage in patient 1 (e) and patient 2 (g). VL viral load, PLT platelet count, Temp body temperature, $W B C$ white blood cell count

Upon hospital admission (day 11), the patient was afebrile, disoriented, anorexic, and very drowsy. His voice was very weak. Examination showed abdominal distension and tenderness, as well as an enlarged and tender left inguinal lymph node. No neck stiffness or abnormal breathing sounds were observed. He could not cooperate with the full physical examination.

Urine tests showed positive for red blood cells (RBCs), WBCs, proteins, and ketones. The platelet count was $29.4 \times 10^{9} / \mathrm{L}$. The ALT level was $207.8 \mathrm{U} / \mathrm{L}$ and AST was $665.2 \mathrm{U} / \mathrm{L}$. The level of lactate dehydrogenase (LDH) was 1569.4 U/L, that of creatine kinase isoenzyme (CKMB) was $47.4 \mathrm{U} / \mathrm{L}$, and hydroxybutyrate dehydrogenase (HBDH) was $974 \mathrm{U} / \mathrm{L}$. The viral load of the SFTSV in blood was $1.90 \times 10^{6}$ copies $/ \mathrm{mL}$. The patient was diagnosed with SFTS, and favipiravir was administered upon hospital admission (day 11).

From the start of favipiravir treatment, the counts for WBCs, lymphocytes, and platelets started to increase, and levels of LDH, CKMB, HBDH started to decrease. Liver function was starting to shift towards the normal range. Melena resolved at day 2 of favipiravir treatment. Neurologic symptoms (disorientation, drowsiness) disappeared,

and abdominal distension resolved at day 3 of favipiravir treatment. Urinary parameters returned to normal 5 days after favipiravir use. Protein in the cerebrospinal fluid (CSF) dropped from $63.5 \mathrm{mg} / \mathrm{dL}$ upon hospital admission to $28.7 \mathrm{mg} / \mathrm{dL}$ at 13 days after initiation of favipiravir treatment. The viral load became undetectable at day 5 of favipiravir treatment (Fig. 1 and Supplementary Fig. 1). The patient was discharged shortly afterward.

The patient was found to be Epstein-Barr virus $(\mathrm{EBV})+$ at day 8 of the disease course with a viral load of $1.24 \times 10^{3}$ copies $/ \mathrm{mL}$. The EBV viral load remained stable throughout the disease course.

\section{Features in both patients}

Tests for the human immunodeficiency virus (HIV) antigen and viral load, hepatitis $\mathrm{C}$ virus (HCV) immunoglobulin (Ig)M and viral load, haemorrhagic fever renal syndrome IgM, tumour markers, syphilis antibody, and rapid plasma reagin were negative in both patients. Both patients showed reduced lymphocyte counts during infection; a more detailed and dynamic lymphocyte profile is shown in Fig. 1e and g. 
Dynamic changes in the immune-cell subtypes were tested in patient 2; all subsets were found to be reduced during infection, and rebounded after favipiravir use except plasmacytoid dendritic cells (pDC) (Supplementary Fig. 2). Although both patients lived in the epidemic area of ticks and SFTSV, they had no clear history or signs of tick bites.

Both patients demonstrated a drop in PLT counts and elevation of viral loads with a much worse course of disease in the second patient (viral load peaked at $1.9 \times 10^{7}$ copies $/ \mathrm{mL}$, PLT nadir at $31 \times 10^{9} / \mathrm{L}$ ). In an untreated patient (as a reference) we observed a viral load as high as $5.01 \times 10^{7}$ copies/ $\mathrm{mL}$, and PLT count as low as $20.3 \times 10^{9} / \mathrm{L}$.

\section{Discussion}

Although the antiviral activity of favipiravir has been tested on small animals, its clinical use has not been reported against the SFTSV or any other member of Bunyavirales. Here, we reported two SFTSV-infected patients treated with favipiravir who reached complete remission.

Both patients were admitted with progressive decreases in the WBC and platelet counts and were diagnosed based on virologic evidence. Patient 2 was at a more advanced stage and showed signs of cardiac injury (increased CKMB level), renal and liver dysfunction, and significant haemorrhagic and neurologic symptoms, which progressed rapidly. The patient had shown early signs of multi-organ failure. The prognosis of patients at this critical stage is usually very poor [5]. After favipiravir treatment, the patients recovered completely.

The favipiravir dose we used was consistent with the recommendation to treat influenza approved by the Japan Food and Drug Administration. In both patients, the viral load dropped remarkably fast directly after the start of favipiravir treatment (Fig. 1). Urine and CSF viral loads were reported to be negative after favipiravir treatment, and blood parameters and symptoms recovered shortly after favipiravir treatment.

Two days after the start of favipiravir treatment in patient 1 , multiple, red itchy papules were observed at his distal extremities, which disappeared after 8 days of symptomatic management. Liver function became abnormal upon favipiravir use and returned to normal after ceasing it. These could be the side effects of favipiravir. Therefore, further investigation is required.

The first patient only showed mild symptoms and signs. At this stage of the disease, it is possible that the patient would recover even without specific antiviral treatment. A well-controlled clinical trial with large patient numbers is required to thoroughly evaluate the efficacy of favipiravir.

\section{Conclusions}

Two patients with SFTS treated with favipiravir showed complete remission. These case studies highlight the potential curative effect of favipiravir.

Funding No research funding is involved in this study.

\section{Compliance with ethical standards}

Conflict of interest All authors declare no conflicts of interests concerning this study.

\section{References}

1. Yun SM, et al. Molecular genomic characterization of tickand human-derived severe fever with thrombocytopenia syndrome virus isolates from South Korea. PLoS Negl Trop Dis. 2017;11:e0005893. https://doi.org/10.1371/journal.pntd.

2. Zhan J, et al. Current status of severe fever with thrombocytopenia syndrome in China. Virol Sin. 2017;32:51-62. https://doi. org/10.1007/s12250-016-3931-1.

3. Mourya DT, et al. Malsoor virus, a novel bat phlebovirus, is closely related to severe fever with thrombocytopenia syndrome virus and heartland virus. J Virol. 2014;88:3605-9. https://doi. org/10.1128/jvi.02617-13.

4. Wang J, et al. Novel phlebovirus with zoonotic potential isolated from ticks, Australia. Emerg Infect Dis. 2014;20:1040-3. https:// doi.org/10.3201/eid2006.140003.

5. Li H, et al. Epidemiological and clinical features of laboratorydiagnosed severe fever with thrombocytopenia syndrome in China, 2011-17: a prospective observational study. Lancet Infect Dis. 2018;18:1127-37. https://doi.org/10.1016/s1473-3099(18)30293 $-7$.

6. Yu XJ, et al. Fever with thrombocytopenia associated with a novel bunyavirus in China. N Engl J Med. 2011;364:1523-32. https:// doi.org/10.1056/NEJMoa1010095.

7. Baranovich T, et al. T-705 (favipiravir) induces lethal mutagenesis in influenza A H1N1 viruses in vitro. J Virol. 2013;87:3741-51. https://doi.org/10.1128/jvi.02346-12.

8. Furuta Y, et al. (T-705 (favipiravir) and related compounds: Novel broad-spectrum inhibitors of RNA viral infections. Antiviral Res. 2009;82:95-102. https://doi.org/10.1016/j.antiviral.2009.02.198.

9. Gowen BB, et al. Modeling severe fever with thrombocytopenia syndrome virus infection in golden syrian hamsters: importance of STAT2 in preventing disease and effective treatment with favipiravir. J Virol. 2017. https://doi.org/10.1128/jvi.01942-16.

10. Guines: priliminary results of the jiki clinical trial to test the efficacy of favipiravir in reducing mortality in individuals infected by ebola virus in guinea[OL]. http://allafrica.com/stories/20150 2241478.html (2015). Accessed 24 Feb 2015

11. Gowen BB, et al. In vitro and in vivo activities of T-705 against arenavirus and bunyavirus infections. Antimicrob Agents Chemother. 2007;51:3168-76. https://doi.org/10.1128/aac.00356-07.

12. Hawman DW, et al. Favipiravir (T-705) but not ribavirin is effective against two distinct strains of Crimean-Congo hemorrhagic fever virus in mice. Antiviral Res. 2018;157:18-26. https://doi. org/10.1016/j.antiviral.2018.06.013. 